\title{
Effects of changes of osteocyte shape on fluid flow and fluid shear stress of loaded bone
}

\section{WeiLun Yu ( $\nabla$ weilun1990@163.com )}

Taiyuan University of Technology https://orcid.org/0000-0002-6253-576X

\section{Xuyang Huo}

Jilin Medical University

\section{Hongliang Li}

Jilin Medical University

\section{Fengjian Yang}

Jilin Medical University

\section{Qiuju Qi}

Jilin Medical University

\section{Xiaogang Wu}

Taiyuan University of Technology

\section{Weiyi Chen}

Taiyuan University of Technology

\section{Research}

Keywords: pressure gradient, osteocyte, Poroelasticity, pore pressure, fluid shear stress, permeability

Posted Date: May 6th, 2021

DOI: https://doi.org/10.21203/rs.3.rs-454657/v1

License: (c) (i) This work is licensed under a Creative Commons Attribution 4.0 International License.

Read Full License 


\section{Effects of changes of osteocyte shape on fluid flow and fluid shear stress of loaded bone}

Yu Weilun ${ }^{1 *}$; Huo Xuyang ${ }^{1 *}$; Li Hongliang ${ }^{1}$; Yang Fengjian ${ }^{1}$; Qi Qiuju¹; Wu

Xiaogang ${ }^{2}$; Chen WeiYi

${ }^{1}$ College of Biomedical Engineering, Jilin Medical University, Jilin, Jilin, China

${ }^{2}$ College of Biomedical Engineering, Taiyuan University of Technology, Taiyuan, Shanxi, China

Corresponding author: * Dr. Yu Weilun, College of Biomedical Engineering, Jilin

Medical University, Jilin 132013, China; Email: weilun1990@163.com.

*Dr. Huo Xuyang, College of Biomedical Engineering, Jilin Medical University, Jilin 132013, China; Email: huoxuyang@aliyun.com. 


\section{Abstract}

Background: Load-induced pressure gradients (PG) result in interstitial fluid flow in bone tissue, which is not only provides sufficient nutrient supply and metabolic pathway for the bone cells, but also enables bone cells to accept external mechanical signals. Methods: We calculate the number and distribution of bone canaliculus around the osteocyte based on the varying shape of osteocyte, and then use these calculated parameters and other microstructure data of bone tissue to estimate the anisotropy permeability of the lacunar-canalicular. Finally, according to the calculated parameters, the poroelastic finite element models of the osteon are established, and the influence of the osteocyte shape on the fluid flow properties of osteon under the axial displacement load is analyzed. Two kinds of boundary conditions are considered on the cement line of osteon, elastic restrained (BC1) and displacement confined (BC2). Results: In the range of parameters we studied, the changes of osteocyte shape (Case1-Case6) make the maximum value of PG, pore pressure (PP), fluid velocity (FV) and fluid shear stress (FSS) 33.36\%, 67.67\%, 8.6\% and $26.6 \%$ larger than the Reference model in $\mathrm{BC} 1$, respectively. And the maximum value of PG, PP, FV and FSS are $65.39 \%, 67.67 \%, 8.4 \%$ and $29 \%$ larger than reference model in $\mathrm{BC} 2$. The maximum $\mathrm{PG}, \mathrm{PP}, \mathrm{FV}$ and $\mathrm{FSS}$ in $\mathrm{BC} 2$ are $96.72 \%$, $95.51 \%, 97.87 \%$ and $97.13 \%$ larger than that in $\mathrm{BC} 1$, respectively. The permeability of elongated osteocyte model (Case 3, Case 4 and Case 6) have a difference of one magnitude order in $\mathrm{X}$ and $\mathrm{Y}$ direction. Conclusion: The changes of osteocyte shape has a significant impact on the degrees of anisotropy for fluid flow and porous media 
of osteon. This model can facilitate better accurately quantifying the fluid flow in lacuna-canalicular system.

Key words: pressure gradient; osteocyte; Poroelasticity; pore pressure; fluid shear stress; permeability

\section{Background}

Cortical bone contains two hierarchical structure of interconnected channels: the large scale is Haversian and Volkmann's canals, and the small scale is lacuna-canalicular. Osteocytes are bathed in the interstitial fluid of the lacuna-canalicular porosity. Mechanical loading drove the free fluid in and out of the pore by causing bone matrix deformation, and the fluid flow shearing osteocyte membranes or inducing cytoskeleton deformation, and thus can activate biochemical responses [1]. Osteocytes are considered the mechanosensors that can sense the mechanical stimulation and transduce them into biochemical signals regulating bone remodelling. Fluid flow play an important role in the mechanosensory system of osteocyte [2]. However, osteocytes are embedded in mineralized extracellular matrix, and direct experimental research is difficult. In order to observe the mechanotransduction and biomechanical behaviours of osteocytes, researchers tried to overcome this problem by establishing mathematical models of fluid flow in bone matrix [3, 4]. Poroelasticity is a well-developed theory for investigating the interaction of fluid and solid phases in bone. Permeability is an important characteristic affecting interstitial fluid flow in bone. Using numerical simulation to explore the fluid flow induced by mechanical loading, the researchers calculate the 
values of PP, FV and FSS in bone by poroelastic model [4-8]. However, these models assume that the permeability of bone is isotropic (osteocytes are spherical), which can't accurately reflect the specific microstructure of the bone (such as the shape, direction and density of lacuna-canalicular system.

In recent years, people are increasingly interested in the spatial characteristics of osteocytes (including morphology and orientation) and the potential relationship between these characteristics and disease [9-12]. Experiments in vitro indicate that the geometry of osteocytes affect its strain response [9, 13]. Carter et al. found that the density, shape and orientation of the osteocytes in the anterior, posterior, medial and lateral femur were significantly different, and the cause may be the local changes in the load [10]. Recent studies have shown that the osteocytes shape of patients with osteoporosis are more irregular, and the bone canalicules are more curved, and the FSS and FV on the osteocytes membrane also change greatly [14]. Age is also an important factor affecting the shape of osteocytes [15]. With the increase of age, the surface area of osteocytes decrease and the shape become flat [16]. The change of osteocyte morphology can lead to anisotropic permeability in bone tissue [17]. In the finite element analysis of poroelasticity, it is necessary to accurately quantify the permeability of lacuna-canalicular to capture the anisotropic fluid flow behavior of bone.

Therefore, in order to more accurately elucidate the specific behavior of intraosseous fluid flow, we developed a poroelastic finite element model based on the microstructure of bone tissue. Firstly, the three-dimensional permeability of bone was 
calculated according to the osteocyte shape. Then, based on the theory of poroelasticity, a finite element model of osteon was established to calculate its fluid flow behavior under axial load, so as to provide a deeper understanding of the mechanism of bone conduction and bone functional adaptation.

\section{Results}

As shown in Fig. 1, the distribution of PG magnitude in the osteon under different boundary conditions at $\mathrm{t}=0.25 \mathrm{~s}$ was plotted. $\mathrm{PG}$ refers to the change of pressure per unit length along the direction of fluid flow. It is one of the main driving forces of fluid flow and other effects (FSS, streaming potential and solute transport) in the osteon $[4,5,18,19]$. Under the axial symmetrical load, the distribution of the PG magnitude in Case 1-Case 6 were quite different from Reference model. The maximum PG of Case 1- Case 6 was $5.49 \mathrm{e} 9 \mathrm{~Pa} / \mathrm{m}$ in $\mathrm{BC}$, and the maximum $\mathrm{PG}$ in Reference model was $3.3 \mathrm{e} 9 \mathrm{~Pa} / \mathrm{m}$. The maximum PG of Case 1 - Case 6 was $33.36 \%$ larger than Reference model. In BC2, the maximum PG value of Case 1- Case 6 was $1.08 \mathrm{e} 10 \mathrm{~Pa} / \mathrm{m}$, and the maximum $\mathrm{PG}$ in Reference model was $6.53 \mathrm{e} 9 \mathrm{~Pa} / \mathrm{m}$. The maximum PG of Case 1- Case 6 was $65.39 \%$ larger than Reference model. This showed that the shape of osteocyte affected distribution of PG magnitude under the same volume of osteocyte. This indicated that when the volume of the osteocyte was the same, the circular osteocyte caused the smaller PP and FV. As shown in Fig 2 and Fig 3, the distribution of PP and FV along on the $y-z$ and $x-z$ planes in Case 1, Case 2 and Case 5 were similar, and these distribution were similar to Reference model. This 
result implied that the elongated osteocytes were more likely to cause the anisotropy of fluid flow in the bone. The maximum value of PG in $\mathrm{BC} 2$ was about twice that of $\mathrm{BC} 1$. This indicated that the right choice of boundary conditions was essential for understanding the fluid flow in bone.

Fig. 1 The magnitude of pressure gradient distribution under different boundary conditions at $\mathrm{t}=0.25 \mathrm{~s}$. $\mathrm{BC} 1$ : elastic restrained. $\mathrm{BC} 2$ : displacement confined.

Fig. 2 and Fig. 3 were the distribution of PP and FV under different boundary conditions, respectively. Due to the different shapes of the osteocyte, the distribution of the PP and FV of Case 1 Case 6 were significantly different from Reference model. From Fig. 2, it could found that the maximum PP value (2.23e5 Pa) in Case1-Case 6 was $67.67 \%$ larger than the Reference model (1.33e5 Pa). In BC2, the maximum PP value (4.36e $5 \mathrm{~Pa}$ ) of Case 1-Case 6 was $67.67 \%$ larger than the Reference model (2.58e5 Pa). The maximum PP in BC2 was $95.51 \%$ higher than that in $\mathrm{BC} 1$. From Fig 3, we can see that, the maximum FV value $(3.76 \mathrm{E}-8 \mathrm{~m} / \mathrm{s})$ of Case 1 - Case 6 was $8.6 \%$ larger than that of Reference model $(3.46 \mathrm{E}-8 \mathrm{~m} / \mathrm{s})$. In $\mathrm{BC} 2$, the maximum $\mathrm{FV}$ value $(7.44 \mathrm{E}-8 \mathrm{~m} / \mathrm{s})$ of Case 1 - Case 6 was $8.4 \%$ larger than that of Reference model $(6.86 \mathrm{E}-8 \mathrm{~m} / \mathrm{s})$. The maximum $\mathrm{FV}$ in $\mathrm{BC} 2$ was $97.87 \%$ larger than that in $\mathrm{BC} 1$.

Fig. 2 The magnitude of PP distribution under different boundary conditions at $\mathrm{t}=0.25 \mathrm{~s}$. BC1: elastic restrained. $\mathrm{BC} 2$ : displacement confined.

Fig. 3 The magnitude of flow velocity distribution under different boundary conditions at $\mathrm{t}=0.25 \mathrm{~s}$. $\mathrm{BC} 1$ : elastic restrained. $\mathrm{BC} 2$ : displacement confined. 
Fig 4 shows the distribution of FSS in the osteon with different shapes of osteocyte at $t=0.25 \mathrm{~s}$. It can be seen that Fig. 3 and Fig. 4 have the similar trend basically in space distribution. In $\mathrm{BC}$, the maximum FSS value of Case 1 - Case 6 model was 3.83 Pa, while the maximum FSS in Reference model was 3.0 Pa. In BC2, the maximum FSS value of Case 1- Case 6 model was $7.55 \mathrm{~Pa}$, while the maximum FSS value in Reference model was 5.85 $\mathrm{Pa}$. In the range of parameters we studied, the change of osteocyte shape would make the maximum FSS value $26.6 \%$ and $29 \%$ larger than that of Reference model in $\mathrm{BC} 1$ and $\mathrm{BC} 2$, respectively.

Fig. 4 The magnitude of FSS distribution under different boundary conditions at $\mathrm{t}=0.25 \mathrm{~s}$. $\mathrm{BC} 1$ : elastic restrained. $\mathrm{BC} 2$ : displacement confined.

\section{Discussion}

This study develops the poroelastic finite element models to investigate the effect of osteocyte shape on fluid flow and FSS in osteon under different boundary conditions. These models are established based on the microstructure of osteon, and simulate interstitial fluid flow arising from mechanical deformation of the osteon and PGs under axial loading representative of physical activity. For modelling purposes, we firstly assumed that the osteons were composed of CPUC, and then we estimated the permeability and porosity of osteon by estimating the number and three-dimensional distribution of bone canaliculus in different shapes of bone lacuna. 
PG refers to the change of pressure per unit length along the direction of fluid flow. This important parameter is often not discussed in previous studies $[4,6,19,20]$. Mechanical loading in osteon occurs on the whole organ level, with compression and tension occurring in different regions driving fluid flow in the lacuna-canalicular system [21]. Maximal PP occurs at the cement line, whereas fluid drainage at the Haversian canal boundary allowed PP magnitudes to be maintained at a lower level. Therefore, a PG across the osteon wall is established, and this PG should be large enough to drive fluid against the trans-cortical pressure difference [22]. In this study, the trans-cortical pressure difference are at least $1.33 \mathrm{e} 5 \mathrm{~Pa}$ and $2.58 \mathrm{e} 5 \mathrm{~Pa}$ in the Reference model in $\mathrm{BC} 1$ and $\mathrm{BC} 2$, respectively. And the PG are sufficient (at least 3.3e $9 \mathrm{~Pa} / \mathrm{m}$ and $6.53 \mathrm{e} 9 \mathrm{~Pa} / \mathrm{m}$ ) to against the trans-cortical pressure difference. As shown in Fig. 1, the PG decreased dramatically away from the Haversian canal. As a result in Fig. 4, relative to the osteocytes close to the Haversian canal, those in the region far away from the Haversian canal experienced a significantly reduced level of FSS.

$\mathrm{PP}$ is an important load inducing phenomenon in the lacuna-canalicular system, which affects the growth, differentiation and chemical transport of osteocyte $[4,5,22$, 23]. The PP changed significantly with the shape of osteocyte. Specifically, the distribution of PP between Case 3, Case 4 and Case 6 in $\mathrm{X}$ and $\mathrm{Y}$ directions is obviously asymmetric, while Case1 Case2, Case5 and Reference models distribution in $\mathrm{X}$ and $\mathrm{Y}$ directions is axisymmetric in both $\mathrm{BC} 1$ and $\mathrm{BC} 2$. This relates to the anisotropy of permeability induced by the change of osteocyte shape. It can be found 
that the permeability of Case 3, Case 4 and Case 6 models are one order of magnitude different in $\mathrm{X}$ and $\mathrm{Y}$ directions, while the permeability of Case1 Case2, Case5 and Reference in $\mathrm{X}$ and $\mathrm{Y}$ directions is little difference. In the $\mathrm{Z}$ direction, the PP of all models have no obvious change. This is because the mechanism of load induced PP makes the fluid flow into the Haversian canal through the lacuna-canalicular, and release the PP [22]. That is, the main fluid flow of osteon is between the cement line and Haversian canal, and there is almost no fluid flow in the $\mathrm{Z}$ direction. As shown in Fig 1, Fig 2, Fig 3 and Fig 4, different boundary conditions have significant effects on the flow behavior in the osteon. The maximum PG, PP, FV and FSS in BC2 is $96.72 \%$, $95.51 \%, 97.87 \%$ and $97.13 \%$ larger than that in $\mathrm{BC} 1$, respectively.

The fluid is allowed passage from Haversian canal and cement line in both BC1 and $\mathrm{BC} 2$. In $\mathrm{BC} 1$, it can be regarded as that some physiological pressure generated outside the osteon can neutralize the PP of the osteon. This condition indicates the PP of the interstitial bone around the osteon is at the same level as the pressure produced by the physiological fluid. In $\mathrm{BC} 2$, the fluid can't across the cement line, which means the cement line is considered to be perfectly rigid and impermeable. Some studies have observed that there are bone canaliculi passing through the cement line $[24,25]$, which indicates that the cement line is permeable and there is fluid exchange between the osteon and the external interstitial bone. From this point of view, BC1 seems closer to the physiological state than $\mathrm{BC} 2$.

Verbruggen et al observed the mean interstitial FV $(\sim 60.5 \mu \mathrm{m} / \mathrm{s})$ and the mean maximum FSS $(\sim 11 \mathrm{~Pa})$ around osteocytes in vivo by applying a load (3000 $\mu \varepsilon$ 
compression and $300 \mathrm{~Pa} \mathrm{PG}$ ) representing strong physiological activity [26]. This result is closer to that of $\mathrm{BC} 2$ in our study, however, the loading in $\mathrm{BC} 2$ represents normal physiological activities. Some studies considered the FSS level required for bone growth is $0.8 \mathrm{~Pa}[26,27]$. FSS in the range of $0.1-2.2 \mathrm{~Pa}$ can increase the production of nitric oxide (NO), prostaglandin (PGE2) and osteopontin[26, 28]. Furthermore, $2 \mathrm{~Pa}$ can increases in intracellular calcium $(\mathrm{Ca} 2+)$, and $0.2-6 \mathrm{~Pa}$ can induce cell response $[7,26]$. Our results $(\sim 3.83 \mathrm{~Pa}$ in $\mathrm{BC} 1$ and $\sim 7.55 \mathrm{~Pa}$ in $\mathrm{BC} 2)$ suggest that the fluid stimulating that osteocytes sensed is sufficiently to create biochemical signals for bone formation, and are similar to values $(\sim 5 \mathrm{~Pa})$ suggested by tracer studies [26, 29]. Interestingly, our findings predict the shape of osteocytes exercise a significant influence on the fluid flow of osteocyte.

One limitation of our research is that the canaliculus is idealized as a straight tube. This study no the effect of curvature of the canaliculus take into account, and in fact, the processes of osteocyte extend through the curved canaliculus from the osteocyte body to the surface of CUPC $[18,26]$. Another limitation is that the osteon is considered to be composed of the identical CUPCs. The shape of the osteocyte in each CUPC may be different, which will lead to the change of local fluid flow. Theoretically, it is necessary to determine the shape of osteocytes in each CUPC, however, it is observed in the experiment that the shape of bone lacuna is similar in a certain region of bone tissue, and such a region is large enough to contain one or several osteons [10]. Therefore, as long as the osteocyte shape in a specific region of bone is determined, the method of this study can be applied to solve the load induced 
FSS and other fluid flow behavior.

\section{Conclusion}

In this study, a method based on the shape of osteocyte is proposed to estimate the anisotropic permeability of lacuna-canalicular system, and the fluid flow in the osteon is described under different boundary conditions according to the calculated permeability. In the range of parameters we studied, the changes of osteocyte shape (Case1-Case6) make the maximum value of PG, PP, FV and FSS 33.36\%, 67.67\%, $8.6 \%$ and $26.6 \%$ larger than reference model in $\mathrm{BC} 1$, respectively. And the maximum value of $\mathrm{PG}, \mathrm{PP}, \mathrm{FV}$ and $\mathrm{FSS}$ are $65.39 \%, 67.67 \%, 8.4 \%$ and $29 \%$ larger than reference model in BC2. The maximum $\mathrm{PG}, \mathrm{PP}, \mathrm{FV}$ and $\mathrm{FSS}$ in $\mathrm{BC} 2$ is $96.72 \%$, $95.51 \%, 97.87 \%$ and $97.13 \%$ larger than that in $\mathrm{BC} 1$, respectively. The permeability of Case 3, Case 4 and Case 6 have a difference of one magnitude order in $\mathrm{X}$ and $\mathrm{Y}$ directions. This indicates that elongated osteocyte are more likely to cause anisotropy of permeability. This study has important reference value for further understanding the mechanotransduction and some bone diseases such as osteoporosis.

\section{Material and Methods}

\subsection{Calculation of osteon permeability based on osteocyte shape}

Fig. 5a is interstitial bone and osteon group. Fig. 5b is a single osteon in Fig 5a. Assuming that the lacuna-canaliculars in Fig. 5b are arranged regularly and the 
distribution of bone canaliculus are uniform, the osteons can be regarded as composing of the cube periodic unit cells (CPUC), which surrounds the osteocyte lacuna (Fig. 5c). Fig. $5 \mathrm{~d}$ is the microstructure of the canaliculus, where $r_{c}$ is the radius of the canaliculus, $r_{o}$ is the radius of osteocyte process, $a_{0}$ is the radius of the fiber matrix around the osteocyte process.

Fig. 5 The hierarchical structure for bone tissue.

Expanding the Weinbaum et al. model to account for the 3-D distribution of the canaliculus, the lacunar-canalicular permeability, klcp, was calculated from the anatomical features of the lacuna-canalicular [8]:

$$
k_{l c p}=\frac{2 \pi n_{\mathrm{i}} a^{4} q^{3}}{\gamma^{3} L^{2}}\left\{A_{1}\left[I_{1}\left(\frac{\gamma}{q}\right)-q I_{1}(\gamma)\right]+B_{1}\left[q K_{1}(\gamma)-K_{1}\left(\frac{\gamma}{q}\right)\right]+\frac{\gamma\left(q^{2}-1\right)}{2 q}\right\}
$$

Where $q$ is the dimensionless ratio between $r_{c}(0.23 \mu \mathrm{m})$ and $r_{o}(0.1 \mu \mathrm{m})\left(q=r_{c} / r_{o}\right)$, $\gamma$ is a dimensionless length ratio between the $r_{c}$ and the square root of the small-scale permeability $\left(k_{p}\right)$ constant for the fluid annulus which is filled with a fiber matrix $\gamma=b / \sqrt{k_{p}}$,and $k_{p}=0.0572 a_{0}^{2}\left(\Delta / a_{0}\right)^{2.377}$. Where $a_{0}$ is the radius of the pericellular fibers ( $5 \mathrm{~nm}$ ), and $\Delta$ is the effective spacing of the fibers of the pericellular matrix ( 7 nm) $[8,17,30]$.

$A 1$ and $B 1$ can be obtained from the following equation:

$$
\begin{aligned}
& A_{1}=\frac{K_{0}(\gamma)-K_{0}(\gamma / q)}{I_{0}(\gamma / q) K_{0}(\gamma)-I_{0}(\gamma) K_{0}(\gamma / q)} \\
& \mathrm{B}_{1}=\frac{I_{0}(\gamma)-I_{0}(\gamma / q)}{I_{0}(\gamma / q) K_{0}(\gamma)-I_{0}(\gamma) K_{0}(\gamma / q)}
\end{aligned}
$$

Where $I_{n}$ and $K_{n}$ denote the first and the second modifications of the Bessel 
function of order $n$, respectively.

$L$ represents the distance between two bone lacunae, which is also the side length of CPUC. It can be obtained from the following formula:

$$
L=\left(\frac{V_{L}}{N_{L a c}}\right)^{\frac{1}{3}}
$$

$V_{L}$ represents unit volume and $N_{L a c}$ represents the number of lacunae per $\mathrm{mm}^{3}$ of bone unit volume. The range of $N_{L a c}$ is $26-90 \times 10^{3}\left(N_{L a c} / \mathrm{mm}^{3}\right)[10,31]$. In this paper, the $N_{L a c}$ value of unit volume was selected $37 \times 10^{3}$, so the value of $L$ was $30 \mu \mathrm{m}$. According to the literature, the average measurement of the total number of bone canaliculi around each bone lacuna is $N=62$ [32]. The morphology of osteocyte was similar to ellipsoid. We used the standard equation of ellipsoid to represent osteocyte.

$$
\frac{x^{2}}{a^{2}}+\frac{y^{2}}{b^{2}}+\frac{z^{2}}{c^{2}}=1
$$

Where the long half axis is $a$, middle half axis is $b$ and short half axis is $c$. The porosity of lacuna-canalicular can be expressed by the following formula:

$$
\varphi=\frac{\mathrm{N} \pi\left(r_{c}^{2}-r_{o}^{2}\right) L c+\frac{4}{3} \pi a b c}{L^{3}}
$$

Where $L_{C}$ is the average length of the bone canaliculi. It was worth noting that because of the fluid exchange between the osteocyte and the bone lacuna, we regarded the whole bone lacuna space and osteocyte body as one pore space. Weinbaum et al. assumed the number of canaliculi passed through the surface of the CPUC in each principal direction were same [8]. However, because of the different of osteocyte shape, the number of canaliculi $\left(n_{i}\right)$ crossing each face of the CPUC was anisotropic [17]. Where $n_{i}$ is the number of canaliculi in the $x, y$, and $z$ directions $(i=x, y$ and $z)$. As 
shown in Fig. 6, the 3-D distribution of the canaliculi was based on the projection surface area of the osteocyte shape. The number of canaliculi in different directions could be measured by the projection area ratio of the osteocyte [17]:

$$
\left\{\begin{array}{l}
\mathrm{n}_{\mathrm{x}}=\frac{1}{2} \frac{S_{x}}{S_{x}+S_{y}+S_{z}} \times N \\
\mathrm{n}_{\mathrm{y}}=\frac{1}{2} \frac{S_{y}}{S_{x}+S_{y}+S_{z}} \times N \\
\mathrm{n}_{\mathrm{z}}=\frac{1}{2} \frac{S_{z}}{S_{x}+S_{y}+S_{z}} \times N
\end{array}\right.
$$

Where $n_{x}, n_{y}$ and $n_{z}$ are the number of canaliculi parallel to the $x, y$ and $z$ axes passing through each face of CUPC, respectively; $S_{x}, S_{y}$ and $S_{z}$ are projected surface areas of bone lacuna in $x, y$ and $z$ directions, respectively [17].

Fig. 6. The projection area of osteocytes along the $\mathrm{x}$ axis $\left(\mathrm{S}_{\mathrm{x}}\right), \mathrm{y}$ axis $\left(\mathrm{S}_{\mathrm{y}}\right)$ and $\mathrm{z}$ axis $\left(\mathrm{S}_{\mathrm{z}}\right)$.

\subsection{The shape of osteocyte}

In order to describe the shape of the osteocyte more clearly, we defined three eigenvalues EV1, EV2 and EV3 (EV1 was the square of the long half axis, Ev2 was the square of the middle half axis and Ev3 was the square of the short half axis) [10]. The shape parameters were then computed for each ellipsoid based upon the resulting three EV. Three ratios of the EV, degree of anisotropy (1-EV3:EV1), degree of elongation (1-EV2:EV1) and degree of flatness (1-EV3:EV2), were modified from studies of particle shape to define the degree of difference. As shown in Table 1 and Fig. 7, several groups of osteocyte with different indicators (anisotropy, elongation 
and flatness) were taken to observe the influence of osteocyte shape on the internal fluid flow. Fig. 7 showed the importance of these three indicators on the shape of osteocyte.

When the three eigenvalues were equal $(\mathrm{EV} 1=\mathrm{EV} 2=\mathrm{EV} 3)$, the shape of osteocyte was spherical (Reference). When EV1 = EV2 $>$ EV3, the shape of osteocyte was flat (Case 1 and Case 2). When EV1 $>$ EV2 = EV3, the shape of osteocyte was elongated (Case 3 and Case 4). When the three eigenvalues are different (EV1 $>$ EV2 > EV3), the shape depended on the extension length and flatness (Case 5 and Case 6).

Fig. 7 Representative cases of osteocyte shapes. The project of osteocyte shapes are shown schematically in (a) the x-y plane, (b) the y-z plane, and (c) the x-z plane.

According to the shape parameters of osteocyte in Table 1, the permeability of Case 1-Case 6 were K1, K2, K3, K4, K5, and K6, respectively. The permeability of the Reference model was isotropic, and its value was $1.05 \times 10^{-20} \mathrm{~m}^{2}$.

$$
\begin{aligned}
& \mathrm{K} 1=\left(\begin{array}{lll}
8.25 \times 10^{-21} & & \\
& 8.25 \times 10^{-21} & \\
& & 1.50 \times 10^{-20}
\end{array}\right) \mathrm{m}^{2}, \\
& \mathrm{~K} 2=\left(\begin{array}{lll}
6.10 \times 10^{-21} & & \\
& 6.10 \times 10^{-21} & \\
& & 1.93 \times 10^{-20}
\end{array}\right) m^{2}, \\
& \mathrm{~K} 3=\left(\begin{array}{lll}
5.74 \times 10^{-21} & \\
& 1.29 \times 10^{-20} & \\
& & 1.29 \times 10^{-20}
\end{array}\right) m^{2}, \\
& \mathrm{~K} 4=\left(\begin{array}{lll}
6.77 \times 10^{-21} & & \\
& 1.28 \times 10^{-20} & \\
& & 1.28 \times 10^{-20}
\end{array}\right) \mathrm{m}^{2},
\end{aligned}
$$




$$
\begin{aligned}
\mathrm{K} 5 & =\left(\begin{array}{lll}
7.31 \times 10^{-21} & & \\
& 7.84 \times 10^{-21} & \\
& & 1.64 \times 10^{-20}
\end{array}\right) \mathrm{m}^{2}, \\
\mathrm{~K} 6 & =\left(\begin{array}{lll}
5.91 \times 10^{-21} & \\
& 1.24 \times 10^{-20} & \\
& & 1.32 \times 10^{-20}
\end{array}\right) \mathrm{m}^{2},
\end{aligned}
$$

Table 1 Geometrical and degree of representative cases

\subsection{Establishment of governing equation and finite element}

\section{model of osteon governing equation}

Due to the periodicity of geometrical configuration, we defined the representative elementary volume (REV) by CUPC. Using poroelasticity theory to describe the fluid flow behavior in osteon was proved to be efficient $[4,5,8,19,20]$. The osteon was illustratesed as a solid-liquid coupling porous elastic material composed of CUPC units in this study, and As shown in Fig. 8, the osteon was regarded as a hollow annular cylinder under cyclic loading in longitudinal orientation.The following governing equations could be used to describe the poroelastic behavior of bone, and nobody forces were taken into account. Constitutive 
laws for the solid matrix material and the saturating fluid were written as [20]:

$$
\begin{aligned}
& \boldsymbol{\sigma}=\mathbf{C} \boldsymbol{\varepsilon}-\boldsymbol{\alpha} p \\
& p=M[\xi-\operatorname{tr}(\boldsymbol{\alpha} \boldsymbol{\varepsilon})]
\end{aligned}
$$

Where $\boldsymbol{\sigma}$ is the total stress tensor, $\mathbf{C}$ is the drained stiffness tensor, $\boldsymbol{\varepsilon}$ is the total strain tensor, $\boldsymbol{\alpha}$ is the Biot's effective stress tensor with the same principal orientations as the compliance tensor, $p$ is the $P P$, Biot modulus is noted $M$ and links the fluid content variation to the pressure in the absence of solid matrix deformations. $\xi_{\text {is }}$ the variations in fluid content and $\operatorname{tr}()$ is the trace operator.

The equilibrium equation is given by:

$$
\rho \ddot{\mathbf{u}}^{s}-\nabla \cdot \boldsymbol{\sigma}=\mathbf{0}
$$

Here, $\rho$ is the total density and $\ddot{\mathbf{u}}^{s}$ is the second derivatives of the displacement. Fluid mass conservation equation is given by:

$$
\frac{\partial \xi}{\partial t}=-\nabla \cdot \mathbf{V}
$$

Fluid flow was calculated by Darcy's law:

$$
\mathbf{V}=-\mathbf{k}\left(\nabla p+\rho_{f} \ddot{\mathbf{u}}^{s}\right)
$$

Here, $\mathbf{V}$ is the velocity vector and $\mathbf{k}$ is the anisotropic permeability tensor that is the textural parameter allowing to quantify the ability of a porous material to transmit fluids through the Darcy's law.

Neglecting body forces, the governing poroelastic equations for anisotropic material in the low frequency range (such as walking, a few Hertz) were given by plugging (8) into (10) and plugging (9) and (12) into (11): 


$$
\left.\begin{array}{l}
\boldsymbol{\alpha} \nabla p=\nabla \cdot(\mathbf{M} \boldsymbol{\varepsilon}) \\
\frac{1}{M} \frac{\partial}{\partial t} p-\nabla \cdot(\mathbf{k} \nabla p)=-\frac{\partial}{\partial t}[\operatorname{tr}(\boldsymbol{\alpha} \boldsymbol{\varepsilon})]
\end{array}\right\}
$$

Considering the low load frequency, the Haversian canal acts as a reservoir to maintain the normal fluid flow in and out. It was assumed that the vascular pores were no longer saturated, so the pressure on the surface of the Haversian canal was set to 0 for reference pressure. The pore size of Haversian canal was much larger than bone canaliculus, so the Haversian canal provided space for fluid pressure to relax when the bone was under mechanical loaded.

Two boundary conditions representing the physiological environment of the osteon were taken into account.

$\mathrm{BC1}$ : the cement line was impermeable and elastic was restrained:

$$
\begin{aligned}
& \left.\sigma_{r r}^{1}\right|_{r=a}=\left.p^{1}\right|_{r=a}=0 \\
& \left.\sigma_{\mathrm{rr}}^{1}\right|_{\mathrm{r}=b}=-\left.p^{1}\right|_{r=b}=0 \\
& \partial p^{1} /\left.\partial r\right|_{r=b}=0
\end{aligned}
$$

BC2: the cement line was impermeable and displacement was constrained:

$$
\begin{aligned}
& \left.\sigma_{r r}^{2}\right|_{r=a}=\left.p^{2}\right|_{r=a}=0 \\
& \left.u_{\mathrm{r}}^{2}\right|_{\mathrm{r}=b}=0 \\
& \partial p^{2} /\left.\partial r\right|_{r=b}=0
\end{aligned}
$$

The FSS experienced by the osteocyte and its processes was obtained by the following equation[22]:

$$
F S S=\frac{8 \mu v_{r}}{d}
$$

Where $d$ is the mean pore diameter: 


$$
d=4 \sqrt{2 \mathrm{~T} \mathbf{k} / \varphi}
$$

Where $v_{r}$ is the interstitial fluid velocity, given by the Dupuit relation:

$$
v_{r}=\frac{T v}{\varphi}
$$

$T$ is the tortuosity of the flow path ( $t=1$ for straight channels) and $v$ is the value of Darcy velocity [22].

\subsection{Establishment and calculation of model}

In this work, COMSOL Multiphysics software was used to investigate the poroelastic behavior of fluid solid interaction in osteon under axial compression load. As shown in Fig. 8a, the osteon was defined as a hollow cylinder that composed of CPUC, and its material and geometric parameters were shown in Table 2.

Table 2 Geometrical and material constants used in osteon model

The compression loads on the top and bottom of the osteon were both represented by a harmonic displacement $(w)$ of amplitude $0.5 \mu \mathrm{m}$ and a frequency $f$, which leaded to the maximum strain loading $\varepsilon=0.001$ at $\mathrm{t}=0.5 \mathrm{~s}$, but the maximum pressure and velocity responses were at $\mathrm{t}=0.25 \mathrm{~s}[6,19]$ :

$$
\left.w\right|_{z= \pm 0.5 \mathrm{~mm}}= \pm 0.00025[\cos (2 \pi f t)-1][\mathrm{mm}]
$$

As shown in Fig. 8(b), the mesh used in the finite element simulation contained 20880 elements and 54277 degrees of freedom.

Fig. 8 Establishment of osteon model (a) and mesh generation (b). 


\section{List of abbreviations}

pressure gradients-PG;

pore pressure-PP;

fluid velocity-FV;

fluid shear stress-FSS.

\section{Declarations}

\section{Ethics approval and consent to participate}

It is not relevant to our work.

\section{Consent for publication}

Not applicable.

\section{Availability of data and material}

The datasets supporting this article have been uploaded as part of the Supplementary Material. All the data and images were calculated by the finite element model. The models are provided in supplementary materials. If reviewers and editors want to repeat the experiment or verify the reliability of experimental modal data, they can directly use the finite element model provided by the author. It is worth noting that COMSOL Multiphysics software is used in this study. 


\section{Competing interests}

No conflict of interest exits in the submission of this manuscript.

\section{Funding}

We received no funding for this study

\section{Authors' contributions}

Weilun Yu designed the study and drafted the manuscript. Xuyang Huo provided much help in the process of drafting the manuscript. Qiuju Qi, Fengjian Yang and Hongliang Li participated in the analysis of the data analysis. Xiaogang Wu and Weiyi Chen provided help in the research ideas. All authors gave final approval for publication.

\section{Ethics approval and consent to participate}

Not applicable.

\section{Acknowledgements}

All the authors listed have approved the manuscript.

\section{Reference}

1. Ganesh T, Laughrey LE, Niroobakhsh M, Lara-Castillo N. Multiscale finite element modeling of mechanical strains and fluid flow in osteocyte lacunocanalicular system. Bone. 2020; 137:115328. 
2. Martin M, Sansalone V, Cooper D, Forwood MR, Pivonka P. Mechanobiological osteocyte feedback drives mechanostat regulation of bone in a multiscale computational model. Biomech. Model. Mechanobiol. 2019; 18(4):1475 - 96.

3. Wu XG, Chen WY. A hollow osteon model for examining its poroelastic behaviors: Mathematically modeling an osteon with different boundary cases. European Journal of Mechanics - A/Solids. 2013; 40:34-49.

4. Wu X, Li C, Chen K, Sun Y, Chen W. Multi-scale mechanotransduction of the poroelastic signals from osteon to osteocyte in bone tissue. Acta Mechanica Sinica. 2020; 36(4):1-17.

5. Jin ZH, Janes JG, Peterson ML. A Chemo-poroelastic Analysis of Mechanically Induced Fluid and Solute Transport in an Osteonal Cortical Bone. Ann. Biomed. Eng. 2021;49: 299-309.

6. Wu X, Chen K, Wang Z, Wang N, Zhao T, Xue Y, Wang Y, Chen W. An analytical poroelastic model for laboratorial mechanical testing of the articular cartilage (AC). Appl Math Mech-Engl. $2018 ; 39(6): 813-28$

7. Wu X, Wang N, Wang Z, Yu W, Wang Y, Guo Y, Chen W. Mathematically modeling fluid flow and fluid shear stress in the canaliculi of a loaded osteon. Biomed. Eng. Online. 2016; 15(2):149.

8. Weinbaum S, Cowin SC, Zeng Y. A model for the excitation of osteocytes by mechanical loading-induced bone fluid shear stresses. J. Biomech. 1994; 27(3):339-60.

9. Wu V, Oers R, Schulten E, Helder MN, Bacabac RG, Klein-Nulend J. Osteocyte morphology and orientation in relation to strain in the jaw bone. Int J Oral Sci. 2018; 10(1):40-7. 
10. Carter Y, Thomas C, Clement JG, Peele AG, Hannah K, Cooper D. Variation in osteocyte lacunar morphology and density in the human femur - a synchrotron radiation micro-CT study. Bone. $2013 ; 54(1): 126-32$

11. Egl A, Mb A, Mbg B, Jms B, Jlb A. Human mesenchymal stem cell morphology, migration, and differentiation on micro and nano-textured titanium. Bioactive Materials. 2019; 4:249-55.

12. Sun T, Yan Z, Cai J, Shao X, Jing D. Effects of mechanical vibration on cell morphology, proliferation, apoptosis, and cytokine expression/secretion in osteocyte - like MLO - Y4 cells exposed to high glucose. Cell Biol. Int. 2020.

13. Ishimoto T, Kawahara K, Matsugaki A, Wang J, Kamioka H, Nakano T. Analysis of Osteocyte Morphology in Terms of Sensation of In Vivo Stress Applied on Bone. In Mater. Sci. Forum. 2014:1265-8.

14. Verbruggen SW, Vaughan TJ, McNamara LM. Mechanisms of osteocyte stimulation in osteoporosis. J. Mech. Behav. Biomed. Mater. 2016; 62(9):158-68.

15. Hemmatian $H$, Bakker AD, Klein-Nulend J, Lenthe G. Aging, Osteocytes, and Mechanotransduction. Current Osteoporosis Reports. 2017; 15(5):1-11.

16. Rolvien T, Schmidt FN, Milovanovic P, Jhn K, Riedel C, Butscheidt S, Püschel K, Jeschke A, Amling M, Busse B. Early bone tissue aging in human auditory ossicles is accompanied by excessive hypermineralization, osteocyte death and micropetrosis. Sci. Rep. 2018; 8(1):1920.

17. Beno T, Yoon Y-J, Cowin SC, Fritton SP. Estimation of bone permeability using accurate microstructural measurements. J. Biomech. 2006; 39(13):2378-87. 
18. Tol A, Roschger A, Repp F, Chen J, Weinkamer R. Network architecture strongly influences the fluid flow pattern through the lacunocanalicular network in human osteons. Biomech. Model. Mechanobiol. 2020; 19(3): 823-840.

19. WeiLun. Y, XiaoGang. W, HaiPeng. C, Yuan. G, ChaoXin. L, YanQin. W, YiXian. Q, WeiYi. C. Study on the biomechanical responses of the loaded bone in macroscale and mesoscale by multiscale poroelastic FE analysis. Biomed. Eng. Online. 2019; 18:122.

20. Chen Y, Wang W, Ding S, Xu W, Chen Q, Xing L. A multi-layered poroelastic slab model under cyclic loading for a single osteon. Biomed Eng Online. 2018; 17(1):97.

21. Tate M. "Whither flows the fluid in bone?" An osteocyte's perspective. J. Biomech. 2003; 36(10):1409-24.

22. Goulet GC, Coombe D, Martinuzzi RJ, Zernicke RF. Poroelastic Evaluation of Fluid Movement Through the Lacunocanalicular System. Ann. Biomed. Eng. 2009; 37(7):1390-402.

23. Gailani G, Cowin S. THEORETICAL ANALYSIS OF THE LEAKAGE THROUGH THE CEMENT LINE OF A SINGLE OSTEON. J. Mech. Med. Biol. 2020; 20(2):950073.

24. Yin D, Chen B, Lin S. Finite element analysis on multi-toughening mechanism of microstructure of osteon. J. Mech. Behav. Biomed. Mater. 2021; 117(2):104408.

25. Nguyen VH, Lemaire T, Naili S. Poroelastic behaviour of cortical bone under harmonic axial loading: A finite element study at the osteonal scale. Med. Eng. Phys. 2010; 32(4):384-90.

26. Verbruggen SW, Vaughan TJ, Mcnamara LM. Fluid flow in the osteocyte mechanical environment: a fluid-structure interaction approach. Biomech. Model. Mechanobiol. 2014; 13(1):85-97. 
27. Han Y, Cowin SC, Schaffler MB, Weinbaum S. Mechanotransduction and strain amplification in osteocyte cell processes. Proceedings of the National Academy of Sciences. 2004; 101(47):16689-94.

28. Bacabac RG, Smit TH, Mullender MG, Dijcks SJ, Loon J, Klein-Nulend J. Nitric oxide production by bone cells is fluid shear stress rate dependent. Biochem. Biophys. Res. Commun. 2004; $315(4): 823-9$.

29. Price C, Zhou X, Li W, Wang L. Real - time measurement of solute transport within the lacunar - canalicular system of mechanically loaded bone: Direct evidence for load - induced fluid flow. J. Bone Miner. Res. 2011; 26(2).

30. Cardoso L, Fritton SP, Gailani G, Benalla M, Cowin SC. Advances in assessment of bone porosity, permeability and interstitial fluid flow. J. Biomech. 2013; 46(2):253-65.

31. Wittig NK, Laugesen M, Birkbak ME, Bach-Gansmo FL, Birkedal H. Canalicular Junctions in the Osteocyte Lacuno-Canalicular Network of Cortical Bone. ACS Nano. 2019; 13(6): 6421-30.

32. Benalla M, Palacio-Mancheno PE, Fritton SP, Cardoso L, Cowin SC. Dynamic permeability of the lacunar-canalicular system in human cortical bone. Biomech. Model. Mechanobiol. 2013; 13(4):801-12. 
Figures

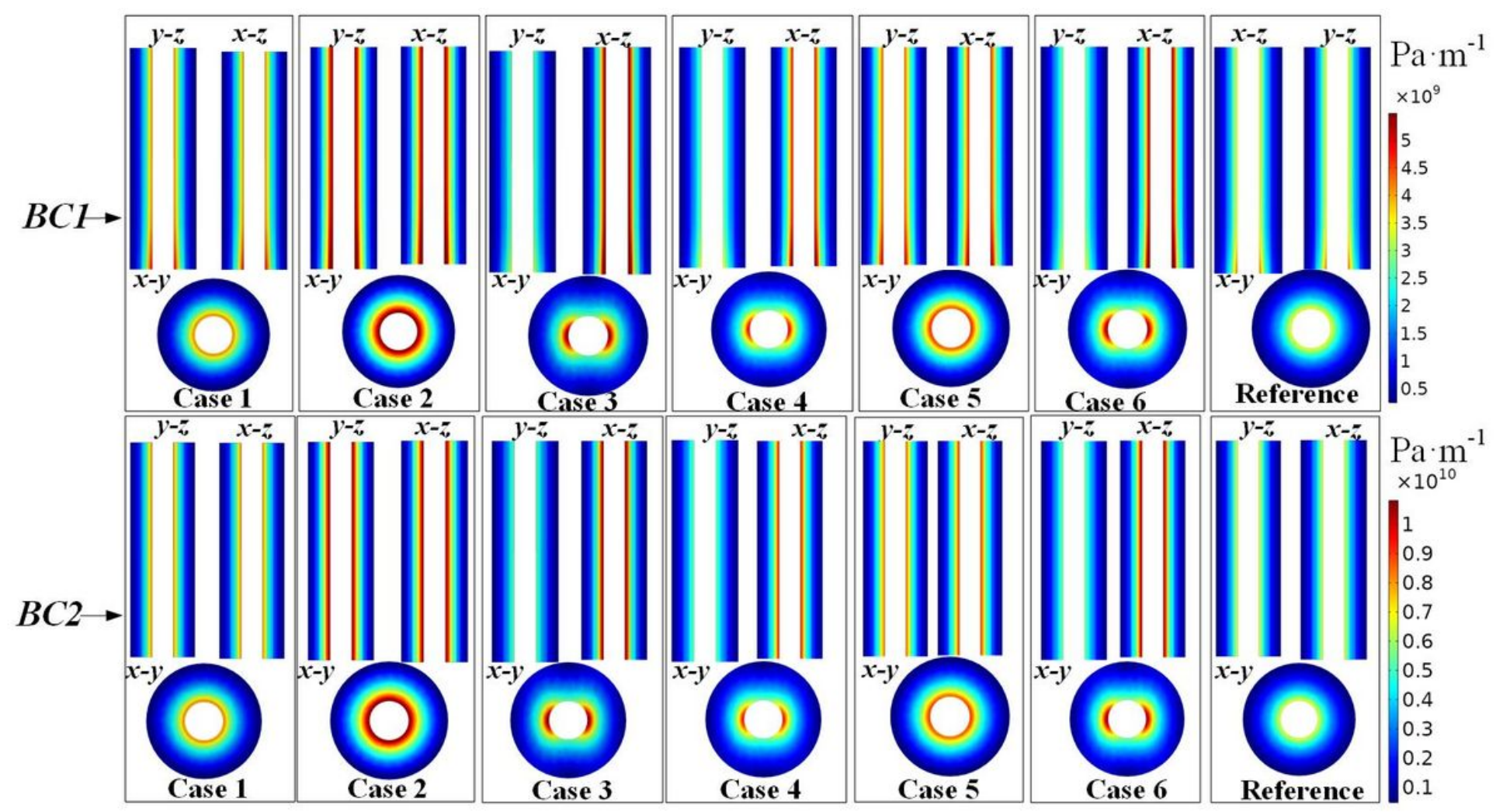

Figure 1

The magnitude of pressure gradient distribution under different boundary conditions at $t=0.25 \mathrm{~s}$. $\mathrm{BC} 1$ : elastic restrained. BC2: displacement confined. 


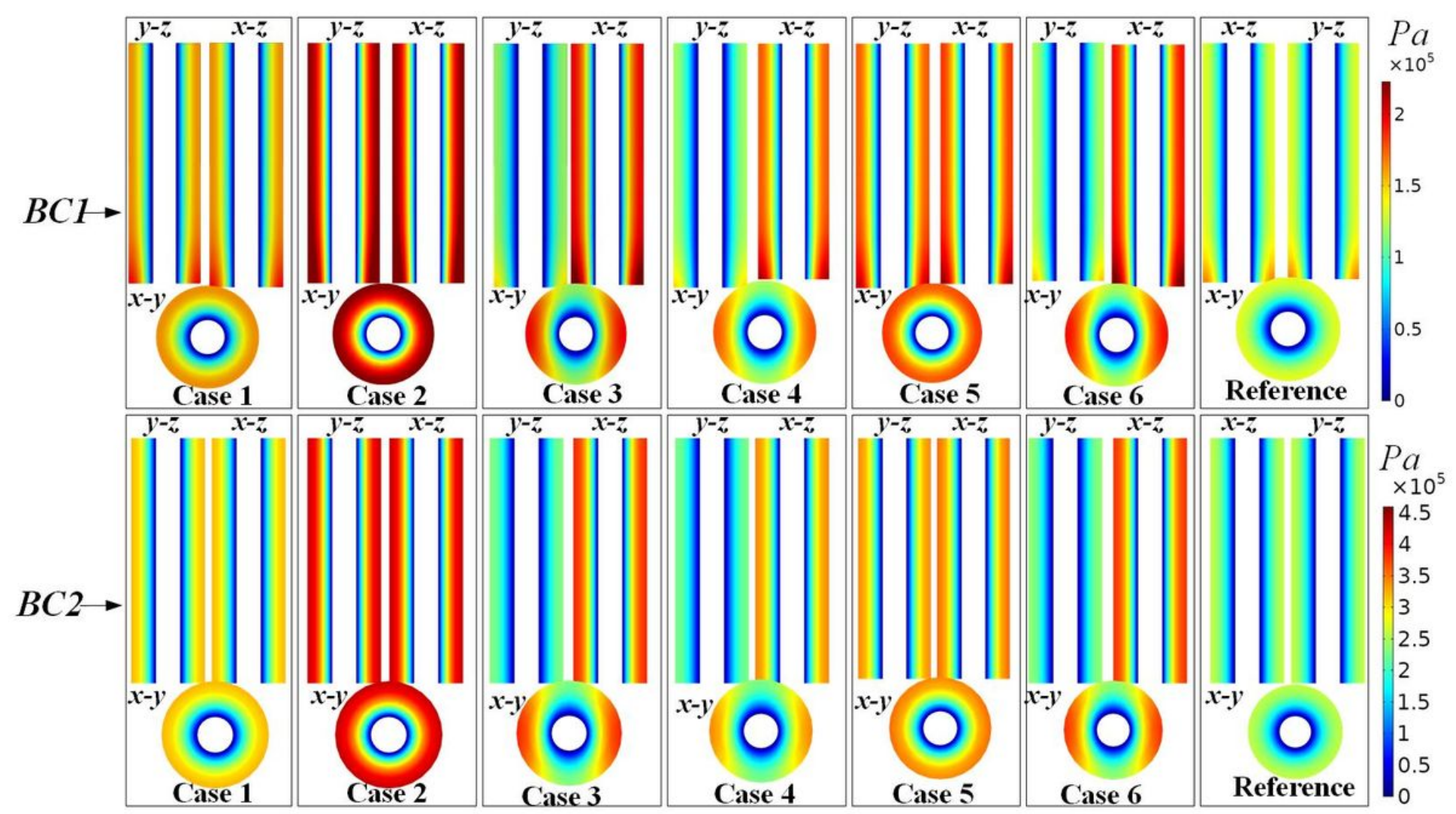

Figure 2

The magnitude of PP distribution under different boundary conditions at $t=0.25 \mathrm{~s}$. BC1: elastic restrained. BC2: displacement confined.

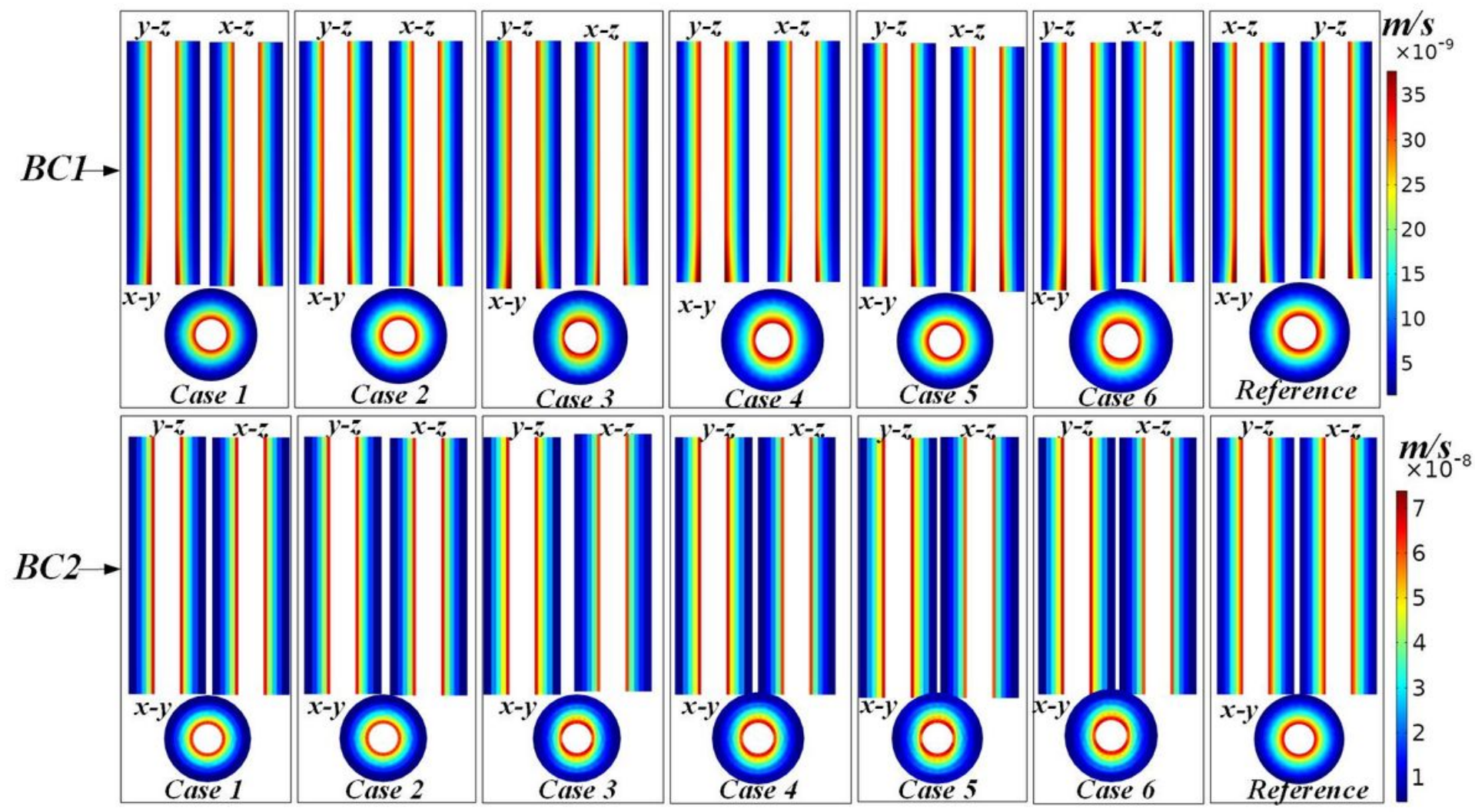


Figure 3

The magnitude of flow velocity distribution under different boundary conditions at $t=0.25 \mathrm{~s}$. BC1: elastic restrained. BC2: displacement confined.

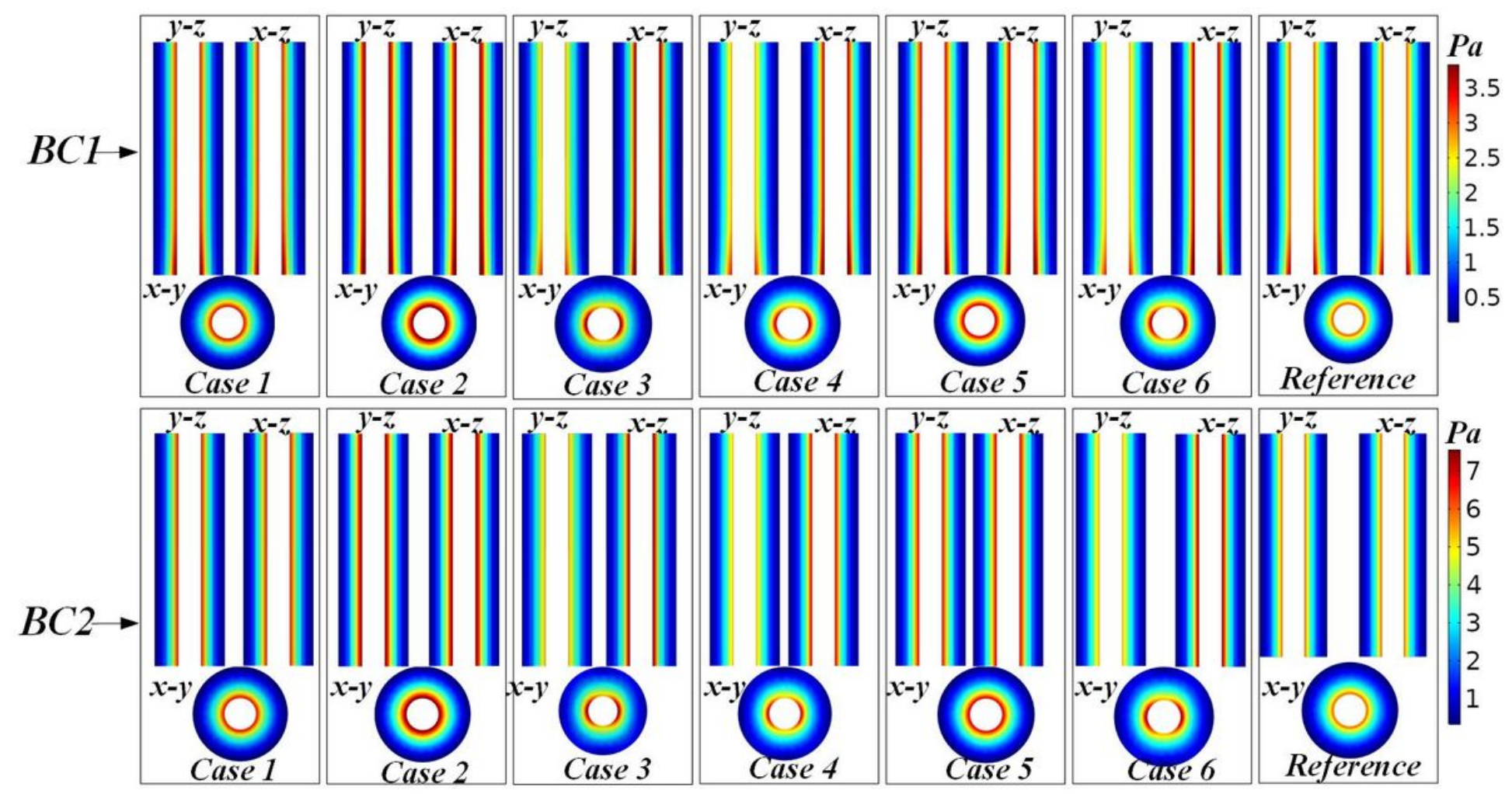

Figure 4

The magnitude of FSS distribution under different boundary conditions at $t=0.25 \mathrm{~s}$. BC1: elastic restrained. BC2: displacement confined. 


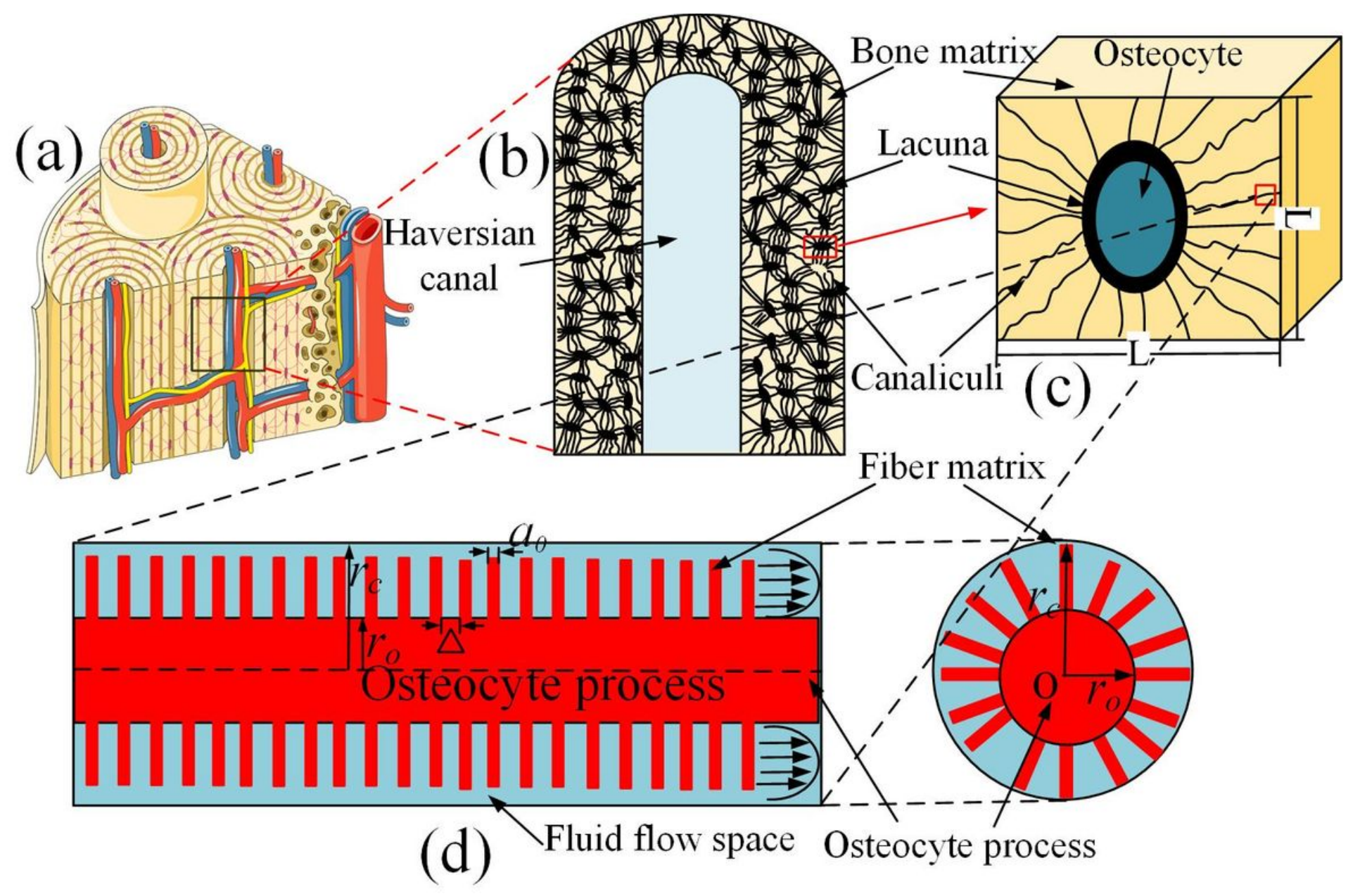

Figure 5

The hierarchical structure for bone tissue.The hierarchical structure for bone tissue. 


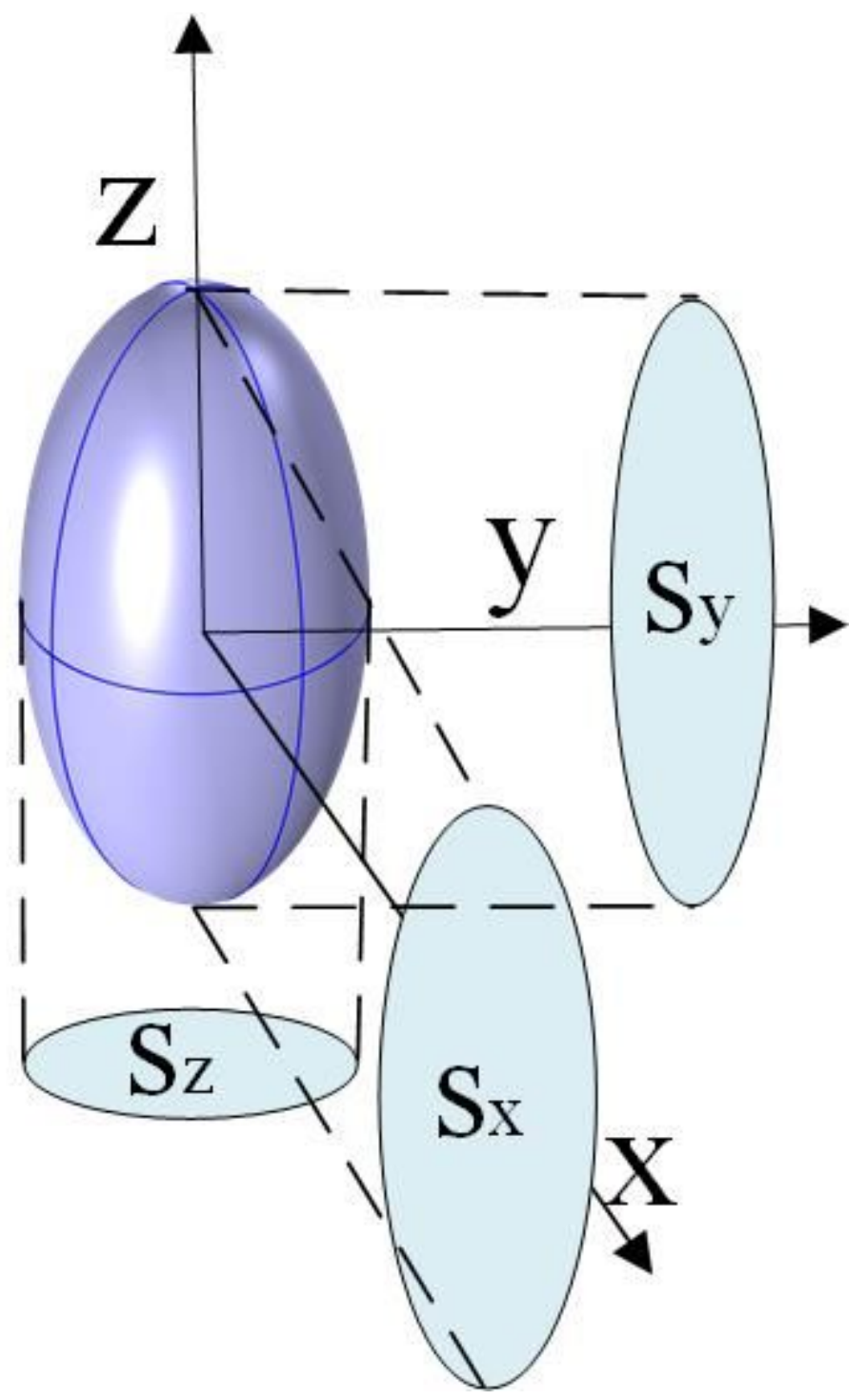

Figure 6

The projection area of osteocytes along the $x$ axis (Sx ), y axis (Sy) and $z$ axis (Sz).

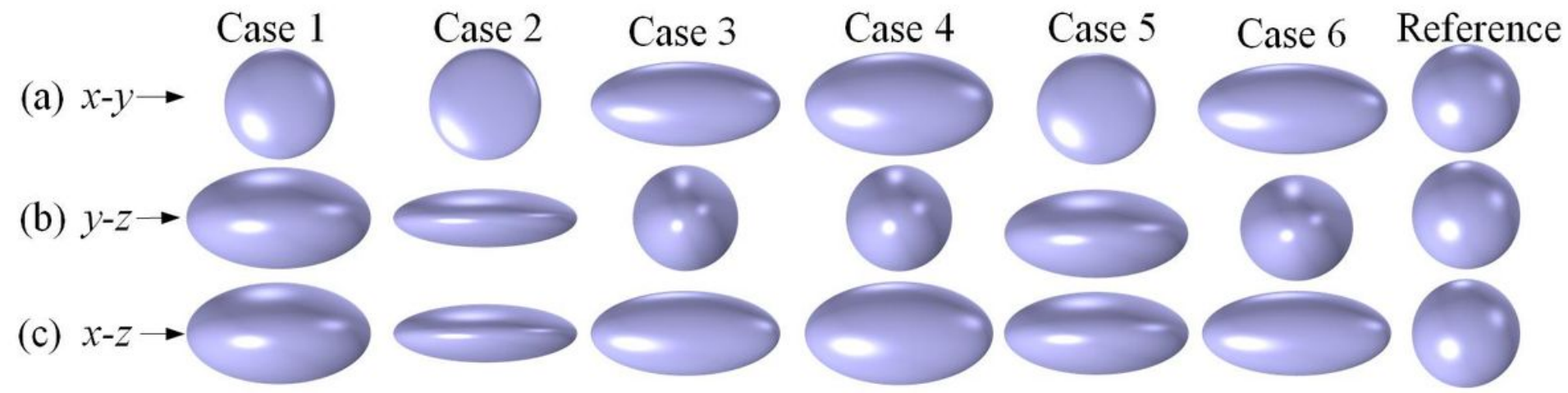

Figure 7

Representative cases of osteocyte shapes. The project of osteocyte shapes are shown schematically in (a) the $x-y$ plane, (b) the $y-z$ plane, and (c) the $x-z$ plane. 

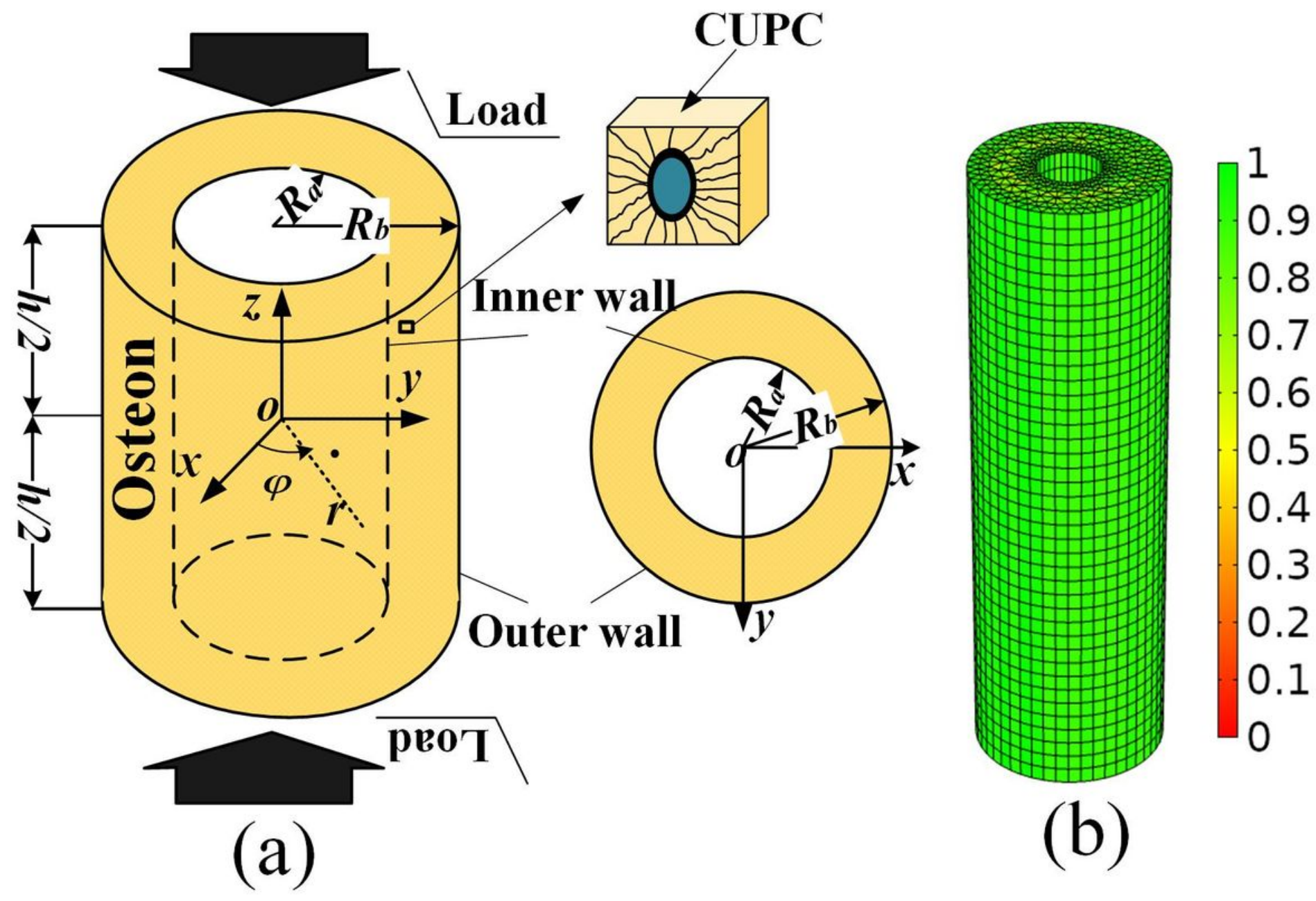

Figure 8

Establishment of osteon model (a) and mesh generation (b).

\section{Supplementary Files}

This is a list of supplementary files associated with this preprint. Click to download.

- Supplementarymaterials.rar 